\title{
Problematique de la Prise en Charge des Personnes Vivant avec le VIH/SIDA en Milieu Rural en Côte d'Ivore: Le cas de la Region du Moronou, Bongouanou
}

\author{
Alexandre Kouassi Ahissan, \\ Docteur en Criminologie, Psycho-criminologue,UFR Criminologie, \\ Université Félix Houphouët Boigny de Cocody, Côte d'ivoire
}

Doi:10.19044/esj.2019.v15n21p150 URL:http://dx.doi.org/10.19044/esj.2019.v15n21p150

\section{Résumé}

Cette étude vise à analyser les prises en charges des personnes vivant avec le VIH/Sida dans la région du Moronou et les difficultés rencontrées pendant ces prises en charge. L'hypothèse stipule que le problème de la prise en charge des malades en milieu rural est lié à l'insuffisance des infrastructures de prise en charge, le manque de personnel formé et l'illettrisme. Dans le cadre de ce travail, trois théories que sont le modèle de la croyance à la santé, la théorie de l'action raisonnée et la théorie du comportement planifié ont été convoquées. Une méthodologie reposant sur l'observation non participante, l'entretien et la recherche documentaire a été utilisée. L'échantillon de la population d'enquête est composé de cinquante quatre individus. Pour l'analyse des données, nous avons opté pour la méthode qualitative. Les résultats de cette étude se présentent sous trois dimensions : les types de prise en charge bénéficiés par les personnes infectées par le VIH/Sida dans la région du Moronou, les difficultés relatives à l'illettrisme des personnes infectées, les difficultés rencontrées par les structures impliquées dans la prise en charge et leur personnel.

Mots-clés: Difficultés, Prise en charge, Personne vivant, VIH/Sida, Milieu rural 


\title{
Problem of Caring for People Living with HIV/AIDS in Rural Environments in Côte d'Ivore: the case of the Moronou Region, Bongouanou
}

\author{
Alexandre Kouassi Ahissan, \\ Docteur en Criminologie, Psycho-criminologue,UFR Criminologie, \\ Université Félix Houphouët Boigny de Cocody, Côte d'ivoire
}

\begin{abstract}
This paper focuses on analysing the treatment of people living with HIV/AIDS in the Moronou region and the difficulties encountered during this treatment. The hypothesis states that the problem of caring for the sick in rural areas is linked to the lack of care infrastructure, the lack of trained staff, and illiteracy. As part of this work, three theories, namely the health belief model, the theory of reasoned action and the theory of planned behaviour, were convened. A methodology based on non-participating observation, interviewing, and document retrieval was used. The sample of the survey population consists of fifty-four individuals. For data analysis, we have opted for the qualitative method. The results of this study are in three dimensions: the types of care provided by people infected with HIV/AIDS in the Moronou region, the difficulties related to the illiteracy of infected people, and the difficulties encountered by the structures involved in care and their staff.
\end{abstract}

Keywords: Challenges, Care, Person living, HIV/AIDS, Rural

\section{Introduction}

\section{Quelques repères théoriques}

Depuis l'apparition des premiers cas du VIH/Sida aux Etats-Unis d'Amérique dans les années 80, la maladie est devenue un problème mondial car elle menace toutes les populations du monde entier. Elle n'épargne personne. Selon Villarreal (2014), Sur les 36,1 millions de personnes infectées par le VIH/SIDA dans le monde, plus de $95 \%$ vit dans les pays envoie de développement. Et dans ces pays, la maladie est plus présente dans les zones rurales que dans les milieux urbains. C'est pourquoi, il y a plus de la population rurale infectée par le VIH/Sida que les personnes vivant en ville. La maladie se propage de manière exponentielle même dans les hameaux les plus reculés(...). La population Africaine ne représente qu'un dixième de la population mondiale. Mais sur les 10 nouveaux cas d'infection déclarés par VIH, 9 sont des Africains. Mieux 83\% des décès sont dus au VIH/Sida en 
Afrique. Et la maladie a dix fois plus meurtrière que la guerre. Dans neuf pays d'Afrique au Sud Sahara, plus de 10 pour cent de la population adulte vit avec le virus.

N'cho et al. ne disent pas le contraire, ils confirment ces données avec les statistiques de la structure mondiale en charge de la gestion de la pandémie. Pour N'cho et al. (2013), en 2010, l'ONUSIDA estimait à 34 millions de personnes infectées par le VIH avec 2,7 millions de nouveaux cas d'infections. L'Afrique subsaharienne est la partie la plus touchée au monde par le virus avec prés de $68 \%$ des personnes infectées dans le monde.

Et la Côte d'Ivoire détient le triste recours du pays le plus touché par la maladie en Afrique Occidentale. En 2005, la séroprévalence était de 4,7\% avec $6,4 \%$ chez les femmes contre $2,9 \%$ chez les hommes. C'est pourquoi, on parle de féminisation du VIH/Sida en Côte d'Ivoire. Pour apporter une réponse efficace à la maladie, des stratégies de lutte sont mises en place. Elles concernent la prévention, le conseil et dépistage, et la prise la prise en charge.

La République démocratique du Congo(RDC) n'est pas épargnée par la pandémie, elle constitue les effets collatéraux de la guerre. Selon AFIA (2016), dans les milieux ruraux du Sud-Kivu, une région de la RDC, la population civile n'est pas en sécurité. Les femmes sont singulièrement touchées avec de nombreux cas de viols, surtout dans le milieu rural. Ces cas sont répertoriés et sont la base même de la propagation des maladies sexuellement transmissibles que sont les IST et le VIH/SIDA. C'est d'ailleurs du fait de l'insécurité que de nos jours, la pandémie ne touche plus singulièrement les villes mais aussi les villages. Le VIH/Sida considéré comme sacré provoque des effets psychologiques, sociaux, et devient parfois une arme de guerre qui détruit un grand nombre de familles qui ne savent comment mener la riposte.

Le sida depuis sa découverte est considéré comme la maladie de la honte. La maladie est aussi liée à la pauvreté. Selon Stepanoff (2010), le problème du VIH/Sida en Afrique est ainsi associé à la pauvreté et à l'insuffisance des infrastructures médicales publiques, le manque de personnel formé, l'illettrisme...Pour lutter contre la pandémie, il faut tenir compte de ces réalités. Le VIH/Sida est un véritable obstacle au développement de l'Afrique. Mieux les acteurs du développement comme les entreprises doivent participer aux efforts de prévention du VIH, au traitement et aux soins nécessaires.

De la méfiance à la critique, de l'ostracisme à la discrimination, du rejet à l'abandon, de la stigmatisation à la spoliation, l'existence d'attitudes hostiles envers les personnes atteintes, nourries par des connotations péjoratives de la maladie, semble être un invariant culturel. 
Cette «maladie sociale » est souvent, pour les personnes infectées et leurs proches, plus difficile à supporter que les signes cliniques de la maladie (Desclaux, 2003).

En zone rurale, les personnes déclarées séropositives rencontrent d'énormes difficultés. Selon Heba(2009), les malades ont peur d'être rejetés s'ils sont identifiés séropositifs. Ils sont entre autre découragés par l'effectif et l'éloignement des structures de soins du VIH et désarmés face à un circuit de prise en charge qu'ils ne maitrisent toujours pas. Au Gabon, les patients séropositifs en milieu rural ont un problème pour accéder aux soins. Il est aussi difficile de convaincre aux malades de suivre convenablement leur traitement.

Toujours pour Heba, Pauvreté et stigmatisation sont des raisons qui poussent les personnes infectées par le VIH/sida à ne pas fréquenter les centres de santé. En ce qui concerne la pauvreté, elle empêche les malades trop démunis à avoir leurs transports pour se rendre en ville et payer les examens médicaux. Ce qui les pousse à renoncer aux soins. Quand les patients ont les moyens, ils ont peur de la stigmatisation liée à la maladie en milieu rural. Ce qui les oblige à renoncer au traitement afin d'éviter de mettre leur vie en danger. Il évoque une autre raison non moins importante qui motive les malades à ne pas fréquenter les services de santé. Il s'agit de la décentralisation qui limite encore les services de santé. Cela entraine l'engorgement des patients dans la majorité des centres de traitement du VIH au Gabon provoquant des longs fils d'attente qui les décourage parfois. Ce qui occasionne des rendez-vous espacés qui les stress souvent.

La décentralisation maintes fois annoncée mais jamais respectée. Les services VIH/Sida sont concentrés dans les grandes villes du pays qui n'arrivent à répondre aux besoins de la population. Ils demeurent donc inaccessibles à la population rurale. Ainsi la stigmatisation et l'engorgement des structures expliquent que certains malades n'hésitent pas à dépenser le peu d'argent qu'ils ont pour parcourir de longue distance afin d'aller se faire soigner loin de chez eux dans la discrétion. Le problème de la régularité des services VIH/SIDA est aussi à la base du découragement des patients et surtout celui de rupture de stock des ARV dans les centres de santé dans les provinces.

La prise en charge des personnes infectées par le VIH/Sida est un véritable problème en Côte d'Ivoire eu égard aux différentes représentations de cette maladie. Cette représentation varie selon les régions et les cultures y afférentes. Elle (la représentation) a un impact considérable sur la prise en charge des malades. Selon Perla (2012), la problématique de la prise en charge des personnes infectées par le VIH/Sida ne date pas d'aujourd'hui. Elle existe il ya longtemps. C'est ainsi que l'individu contaminé par le VIH/Sida est un sujet de droit, qui a besoin d'être protégé, eu égard aux nombreux problèmes 
auxquels il est confronté. Le problème est que beaucoup d'individus ont des informations erronées sur la sexualité. Ce qui fait qu'ils contractent facilement les maladies sexuellement transmissibles. Ceci est d'autant plus préoccupant que quand ces derniers vivent dans un pays où la sexualité est encore un tabou au sein des familles.

La prise en charge et le soutien sont fondés sur une préoccupation réelle pour le bien être des autres et de soi-même. Les personnes infectées par le $\mathrm{VIH} /$ sida, leur famille et leur communauté ont besoin d'un accompagnement pour affronter aux difficultés de la maladie afin d'y faire face. Le but de la prise en charge dans le domaine du VIH est d'améliorer la qualité de la vie non seulement des personnes infectées par le VIH/sida et leur famille et surtout celle de leur communauté. La prise en charge est autant plus importante dans la mesure où il consolide les efforts déployés pour éviter que la maladie ne se propage (ONUSIDA, 2003). La prise en charge doit être totale intégrant tous les aspects, raison pour laquelle Thani (2014) attire l'attention de la ministre française des affaires sociales et de la santé sur la problématique de lutte contre le sida et de prise en charge des personnes vivant avec le virus de l'immunodéficience humaine(VIH) à Mayotte. Selon lui, Il n'existe pas dans cette localité, d'actions médiatiques de grande envergure sur la prévention du VIH/Sida et de manière globale, des infections sexuellement transmissibles (IST). L'ignorance, le tabou et le poids de la stigmatisation n'incitent pas la population à se protéger ni les personnes infectées à se faire dépister ou soigner. Toujours pour Thani, les personnes vivant avec le VIH ne bénéficient pas de la même qualité de soins qu'en France métropolitaine, ni même à la Réunion. Il n'existe pas de médecins spécialistes, pas d'hôpitaux de jour, pas d'éducation thérapeutique, pas de prise en charge pluridisciplinaire. La seule association mahoraise recensée ne peut répondre à toutes les demandes d'accompagnement formulées par les populations de cette location eu égard à la forte demande formulée par les services sollicités.

Pour une prise en charge complète, il est donc nécessaire aux patients d'ajouter la prise en charge thérapeutique. Celle-ci implique l'utilisation des antirétroviraux qui sont des médicaments qui retardent l'évolution de la maladie. La prise des ARV n'est pas sans conséquence. C'est à ce niveau que l'observance est indispensable. Selon Yeni (2006), les traitements antirétroviraux sont complexes et difficiles à prendre et on comprend alors tout l'enjeu que représente l'observance dans cette pathologie devenue chronique. Les résultats thérapeutiques peuvent en effet être compromis quand le malade ne suit pas les prescriptions médicales. Mieux, l'horaire et l'espacement des prises des médicaments très sont importants pour les malades. La réduction de fréquence des prises et du nombre de médicaments pris et surtout le non dose prescrite jouent sur l'efficacité des médicaments. D'autres aspects des antirétroviraux ont été révélés. Il s'agit de la toxicité, de la tolérance du 
médicament contre le VIH/Sida et surtout de l'acceptabilité du traitement. Selon Delfraissy (2002), en effet la prise en charge de l'infection par le VIH s'inscrit dorénavant autour de la gestion d'une maladie sur une longue période de traitement. A côté de l'efficacité des antiviraux, les problèmes de tolérance, de toxicité et d'acceptabilité du traitement sont devenus une préoccupation importante tant pour les malades que pour les équipes médicales. La prise en charge médicale d'une personne infectée par le VIH/Sida doit être individualisée, multidisciplinaire, faisant intervenir toute l'équipe médicale. Les complications liées aux traitements antirétroviraux sont de nos jours un problème important. Le bilan des risques cardiovasculaires et une attitude préventive doivent faire partie de la prise en charge. Le problème de la prévention doit être abordé par le corps médical comme une préoccupation majeure. Cela concerne aussi la prise en charge de la sexualité des patients suivis. Prendre en compte la maladie sur le long terme avec ses nouveaux aspects, sans oublier les stratégies thérapeutiques comme la co-infection, pour faciliter les complications liées aux traitements. Ce sont les trois objectifs prioritaires pour les prochaines années, en attendant la venue de nouvelles thérapies.

Dans la prise en charge des personnes infectées par le VIH/sida, le malade joue un rôle très important. C'est ce qui est mentionné par les différents auteurs. Pour ces auteurs, le malade est centre de la prise en charge. C'est pour quoi sont apport reste déterminant pour la réussite de la prise en charge. Ainsi, l'implication des personnes infectées par le VIH/Sida dans la prise en charge médicale est présentée comme une meilleure pratique de soins (OMS, 1998 ; ONUSIDA, 1997a, 1997b, 2000) et aussi comme un élément important dans l'amélioration de leur accès aux soins (Eboko et al., 2010). On assiste à une nette évolution du rôle du malade dans sa propre prise en charge. Parsons (1951) dans le parcours de soins en milieu hospitalier : il est passé du rôle de «patient » (Balint, 1957) à celui de «client » (Mounsadé, 2012 ; MC Laughlin, 2009), et d' « usager de l'offre de soins » à «patient expert» (Pierron, 2007). Le « patient expert » a d'abord eu un rôle informatif (Epstein, 2001) puis celui d'acteur qui impulse une politique de santé (Barbot, 2002). En effet, comme l'écrit (Weller, 2003, p.38) : «Qu'il s'agisse de prévention, d'écoute sociale ou de recherche thérapeutique, des dispositifs inédits se sont imposés et ont contribué à donner aux malades une place et un rôle plus importants qu'il ne leur était reconnu pour d'autres pathologies ». Dans le contexte camerounais, l'expérience du malade est présentée dans la lutte contre le sida comme une stratégie d'amélioration de la qualité de soin. Le rôle du patient expert comprend alors essentiellement une activité de témoignage et un partage d'expériences vécues. Ici, il s'agit des personnes infectées au VIH qui ont surpassé les représentations sociales du VIH, qui ont accepté leur sérologie et surtout qui ont été promu à une bonne observance thérapeutique. 
Cette stratégie a conduit au recrutement de personnes infectées par le VIH/Sida dans le parcours de soins hospitaliers (Plan Stratégique National de Lutte contre le sida, 2000) et à l'opérationnalisation d'une politique de regroupement en associations. Une intervention presque exclusive des mouvements associatifs de lutte contre le sida - du suivi psychosocial des personnes infectées dans les structures de santé. Ceci a fait proliférer le nombre d'associations de manière considérable (de quatre entre 1991-1999, à une centaine dans les années 2000). Et chacune des associations étant en quête d'une intervention dans l'espace hospitalier. Cet investissement de l'hôpital par les associations s'est accentué avec les projets de développement initiés dans la lutte contre la pandémie par le Fonds mondial et les ONG internationales.

Cette revue de littérature a permis de mettre en relief la qualité des écrits réalisés sur la notion de la prise en charge des personnes vivant avec le VIH/Sida en milieu rural. Au regard des recherches effectuées dans ce domaine, il se dégage un ensemble de problèmes liés à la prise en charge des personnes infectées par le VIH/Sida. Aussi cette étude a-t-elle pour objectif d'analyser les différentes prises en charges reçues par les personnes vivant avec le VIH/Sida dans la région de Bongouanou et les difficultés rencontrées. Cette préoccupation laisse apparaitre une question à savoir, quelles sont les difficultés rencontrées dans les différentes prises en charge des personnes infectées par le VIH/sida dans la région du Moronou ? Répondre à une telle question, revient à analyser les différentes prises en charge que bénéficient les malades du Sida et les difficultés rencontrées. Ce travail de recherche tente de vérifier l'hypothèse opératoire suivante: Le problème de la prise en charge des malades en milieu rural est lié à l'insuffisance des infrastructures de prise en charge, le manque de personnel formé et l'illettrisme.

Dans le cadre de notre travail, trois théories du changement de comportement seront utiles à savoir : le modèle de la croyance à la santé, la théorie de l'action raisonnée et la théorie du comportement planifié.

Le modèle de la croyance à la santé est l'une des premières théories du comportement en lien avec la santé. Le health belief model a été développé dans les années 1950 par les psychologues sociaux que sont Irwin M. Rosenstock, Godfrey M. Hochbaum, S. Stephen Kegeles, et Howard Leventhal du U.S. Public Health Service pour mieux comprendre l'échec généralisé des programmes de dépistage de la tuberculose. Le health belief model a été appliqué pour prévenir une grande variété de comportements liés à la santé, tels que les moyens de dépistage pour la détection précoce des maladies asymptomatiques afin de recevoir éventuellement des soins. Plus récemment, le modèle a été appliqué pour comprendre les réponses des patients aux symptômes d'une maladie, au respect des traitements médicaux, 
aux habitudes de vie (par exemple, les comportements sexuels à risque), et les comportements liés aux maladies chroniques, ce qui peut nécessiter une régularité de comportements sur le long terme, en plus du changement du comportement initial. Les modifications apportées au modèle ont été faites vers 1988, en y associant des nouvelles informations liées aux concepts psychologiques de l'auto-efficacité dans la prise de décision et du comportement. Le Health Belief Model (HBM) est un modèle de psychologie servant à expliquer et prédire les comportements en lien avec la santé, et singulièrement l'utilisation des services de santé. Le health belief model reste l'une des théories les plus connues et les plus utilisées dans la recherche sur les comportements de santé. Il suggère que les croyances des gens au sujet des problèmes de santé, des avantages et obstacles perçus, et de l'auto-efficacité expliquent l'engagement ou non du comportement de promotion de santé ; un signal doit être présent pour déclencher le comportement. Cette théorie a permis de comprendre que les individus, une fois dépistés positifs au VIH/Sida doivent adopter un nouveau comportement pour suivre leur traitement. Cette nouvelle vie permettra d'adopter la maladie. Si non tout ce qu'on entreprend est voué à l'échec. Il faut aussi une collaboration parfaite avec les institutions sanitaires et ce, sur du long terme. Cette théorie n'est suffisante pour rendre compte du phénomène. Ainsi, après le modèle de la croyance à la santé, nous allons voir la théorie de l'action raisonnée $(1967,1980)$. En se basant sur ces développements, Fishbein et Ajzen unirent leur force afin de créer une méthode visant à prédire les comportements humains. Pour ce faire, ils assumèrent que les hommes étaient habituellement rationnels et logiques, et qu'ils utilisaient de façon systématique l'information disponible. Selon le raisonnement des deux chercheurs, les gens considèrent les conséquences de leurs actes avant de décider s'ils adoptent ou non un certain comportement (Ajzen \& Fishbein, 1980; cités dans Mc Cormack Brown, 1999a). Ils en sont venus à développer une théorie qui, selon eux, pouvait prédire et comprendre les comportements et les attitudes. Cette théorie, qu'ils nommèrent la Théorie de l'action raisonnée, suggère que l'intention d'agir est un indicateur du comportement plus important que les attitudes. Selon cette théorie, les attitudes interagissent avec les normes subjectives pour influencer l'intention d'agir, qui elle, détermine le comportement. Cette théorie nous enseigne qu'avant de poser un acte, il faut mesurer les conséquences de cet acte. C'est pourquoi les malades doivent être vigilants dans leur prise de décision, sinon, cela leur pourrait être fatal.

Comme elle a déjà été décrite dans ce qui précède, nous analyserons davantage une autre théorie que ces mêmes auteurs ont développée ultérieurement, c'est-à-dire la Théorie du comportement planifié (1988), qui vise à complémenter et parfaire leur première théorie élaborée. En effet, après avoir découvert des lacunes dans la structure de celle-ci, Ajzen et Fishbein ont 
modifié la Théorie de l'action raisonnée en y ajoutant un troisième élément : le contrôle comportemental perçu. Ce concept est défini par la croyance de l'individu dans l'idée qu'il serait facile ou non d'adopter un certain comportement. Cet élément vise les situations dans lesquelles les gens possèdent peu de contrôle sur leurs comportements et leurs attitudes (Mc Cormack Brown, 1999a). L'idée principale qui découle de cette nouvelle théorie se résume ainsi : les individus ne seront pas capables de développer une forte intention d'agir et de se comporter d'une certaine façon s'ils croient ne pas avoir les ressources nécessaires ou les opportunités pour y arriver, et ce même s'ils possèdent des attitudes favorables envers le comportement en question et s'ils estiment que les membres de leur entourage approuveraient le comportement. Les implications éducatives de cette théorie s'avèrent importantes, car elles peuvent servir à orienter le curriculum et les programmes éducatifs en ce sens, en fournissant aux élèves des opportunités de développer leur intention d'agir, en les plaçant dans des situations réelles et signifiantes (Hanna, 1995). Ici, la théorie ajoute que pour prendre une décision, il faut avoir les moyens et les ressources nécessaires de pouvoir réussir. Les malades sont responsables de leurs propres actes.

\section{Methodologie}

\section{Site et participants}

Cette étude s'est déroulée dans la région du Moronou composée des départements de Bongouanou, de M'batto et d'Arrah. Cette région compte trois hôpitaux généraux, huit centres de santé urbains et un dispensaire foyer de charité. Dans cette région, on y trouve deux ONG de lutte contre le VIH/Sida (Tous pour le toukpè) et (ACP2H : Action contre la pandémie VIH et autres handicapes). En effet, la région du Moronou est située au Centre-Est de la Côte d'Ivoire. Elle est constituée de quatre départements qui sont le département de M'batto, département d'Arrah et le département de Bongouanou (chef lieu de région). La région de Bongouanou est située à $200 \mathrm{Km}$ d'Abidjan. Elle est l'ancienne boucle du cacao. La population est composée d'autochtones (Agni moroffoué), d'allochtone (Baoulé, Dioula) et originaire de la CEDEAO (Burkinabé, Béninois, Togolais, Malien, Ghanéen...) à la recherche d'un mieux être. Bongouanou est une région cosmopolite dont le brassage culturel est très fort. Ce qui fait que le sida est diversement représenté.

La population de cette étude est constituée du personnel administratif et médical des hôpitaux et autres centres de santé sans toutefois oublier des responsables d'ONG, des médecins, des infirmiers, des sages-femmes, des aides soignants, des assistants sociaux, des personnes infectées par le VIH/Sida et la population témoin. L'échantillon d'enquête estimé à 54 personnes reparties de manière suivante : huit (8) agents de santé (2 médecins 
et 4 infirmiers, 2 sages-femmes et 2 aides-soignants), trois (3) assistants sociaux, six(6) responsables (deux coordonnateurs, deux superviseurs, deux chargés de suivi-évaluation), vingt (20) personnes infectées par le VIH/Sida, quinze (15) personnes de la population témoin. Cet échantillon a été constitué autour des personnes volontaires et surtout disponibles.

La détermination de l'échantillon d'enquête s'est faite sur la base de deux techniques d'échantillonnage que sont la technique par choix raisonné et celle en boule de neige. Ces techniques d'échantillonnage non probabiliste ont la particularité d'une part de laisser la latitude au chercheur de s'orienter vers les personnes qu'il désire inclure dans son échantillon, en tenant compte de certaines spécificités et d'autre part de s'orienter vers d'autre cible recommandée. Cette dernière technique a été particulièrement utilisée chez les personnes vivant avec le VIH/Sida en raison de l'omerta que constitue la question du VIH/Sida. Notons que le choix des personnes interrogées présente des caractéristiques en lien avec la compréhension de l'objet d'étude. Ces techniques non-aléatoire ont été utilisées dans un souci de représentativité de la population cible et de réduction des biais méthodologiques.

\section{Protocole et matériel de recueil de données}

La collecte des informations relatives à l'objet d'étude s'est heurtée tout au long de son déroulement à un problème de recueil des données tant le sujet d'étude sur le VIH/Sida est tabou et fait l'objet d'un silence chez des personnes infectées par le VIH/Sida dans la localité de Bongouanou.

Dans le cadre de ce travail, nous avons eu recours aux outils de recueil de données que sont la recherche documentaire, l'entretien à questions ouvertes et l'observation non participante. La recherche documentaire se justifie dans cette étude par la nécessité d'approfondir l'état des connaissances et savoirs sur le sujet à l'étude, afin d'enrichir la littérature et de mieux circonscrire le travail en se focalisant sur les travaux des prédécesseurs. Quant à l'entretien soumis à la population d'enquête, il a permis d'obtenir des informations sur le sujet qui fait l'objet de notre recherche. En effet un entretien à questions ouvertes individuel a été réalisé auprès des enquêtés notamment la population cible que sont les personnes vivant avec le VIH/sida. Ainsi, du fait de la sensibilité du sujet qui au demeurant est tabou, nous avons rassuré les enquêtés de la confidentialité et de l'anonymat des répondants. Il faut aussi retenir que dans le cadre de l'élaboration de ce travail, nous avons utilisé des initiales des nom et prénom des enquêtés pour garder le caractère anonyme du travail. Ce sont donc 52 entretiens individuels réalisés durant la période de l'enquête. Il a pour avantage de permettre aux individus interrogés de développer leurs pensées.

Cette étude de type empirique, a conduit à opter pour une observation non participante du fait de son champ d'étude. Elle a servi à présenter et 
reconstituer objectivement la réalité du terrain en rapport avec le sujet d'étude. Elle a donné l'occasion de rencontrer et d'échanger avec les enquêtés dans la région de Bongouanou et d'apprécier de visu les différentes prises en charge bénéficiées par les malades. Cette technique a permis de corriger les items qui pourraient rebuter les acteurs particulièrement les malades suite à une préenquête. En effet, malgré les précautions et les assurances données, plusieurs personnes infectées par le VIH/sida et la population témoin ont refusé de participer à l'entretien du fait de sa sensibilité.

\section{Analyse des données}

L'analyse des données recueillies s'appuie sur une analyse qualitative notamment l'analyse de contenu afin de faire ressortir le sens des discours et des réponses apportées à l'entretien. Elle permet de mettre en relief les préoccupations des malades et des médecins pour une meilleure prise en charge. Cette approche qualitative exploratoire accorde une place centrale à l'acteur social qui vit la réalité sociale étudiée. En procédant ainsi, la méthode qualitative a permis de mieux comprendre le phénomène.

\section{Resultats}

Les résultats obtenus dans cette étude se présentent sous trois dimensions: les types de prises en charge bénéficiés par les personnes infectées par le VIH/Sida dans la région du Moronou, les difficultés relatives à l'illettrisme des personnes infectées, les difficultés rencontrées par les structures impliquées dans la prise en charge et leur personnel.

\section{Types de prises en charge bénéficiés par les personnes infectées par le VIH/Sida dans la région du Moronou}

Les personnes vivant avec le VIH/Sida dans la région du Moronou bénéficient de deux types de prise en charge à savoir la prise en charge psychosociale et la prise en charge médicale ou thérapeutique.

\subsection{Prise en charge psychosociale}

La prise en charge prise psychosociale (accompagnement psychosocial) est composée de deux types de prise en charge : la prise en charge psychologique et la prise en charge sociale. Dans la région du Moronou l'accompagnement psychologique est assuré généralement par les assistants sociaux, les infirmiers, les sages-femmes, les aides soignants, les médecins et les responsables d'ONG. En effet, cette prise en charge commence dès l'annonce du statut sérologique de la personne. Cette annonce se fait généralement par un médecin. "Avant d'annoncer le statut sérologique d'un individu, il ya une préparation psychologique à faire. L'annonce ne doit pas se faire de manière brutale. Nous devons le préparer à accepter la nouvelle. 
Quelque soit la préparation de l'individu, il reçoit un choc». avoue K.N., médecin. Face à cette situation, le malade doit être fort psychologiquement. C'est d'ailleurs ce qui savoir un patient, A.D. en ces termes: " quand le médecin m'a annoncé ma séropositivité, j'étais psychologiquement abattu. Il a fallu que le médecin me remonte avec des paroles réconfortantes en me disant que ce n'était pas une fatalité et que je pouvais vivre le plus longtemps possible. Finalement, je me suis ressaisi ». Après l'annonce du statut sérologique de l'individu et qu'il est déclaré séropositif, il revient aux ONG (organisations non gouvernementales) d'assurer l'accompagnement psychologique du patient en lui donnant des conseils d'usage afin d'accepter son état. Le soutien psychologique peut favoriser également une meilleure intégration du malade dans la société.

Concernant la prise en charge sociale, elle est dévolue aux ONG. Elle se manifeste par l'achat des médicaments des patients. Les malades souffrent généralement de maladies opportunistes, et puis qu'ils sont en zone rurale, ils sont limités financièrement. Leur dernier recours, c'est les ONG. C'est que $P$. E. responsable d'O.N.G explique : "vous savez, nous sommes en zone rurale et la majorité de nos patients vivent en dessous du seuil de la pauvreté. En effet quand ils reçoivent des ordonnances et qu'ils n'arrivent pas à payer, nous constituons leur dernier recours. Donc, ils nous sollicitent souvent pour payer leurs ordonnances. Vu leur état, nous nous exécutons pour les satisfaire ». Mais en ce qui concerne la prise en charge médicale ou thérapeutique proprement dite, elle échoit au corps médical.

\subsection{Prise en charge médicale ou thérapeutique}

A l'instar aux autres malades de Côte d'Ivoire, la population de Moronou bénéficie d'une prise en charge médicale ou thérapeutique. Elle permet aux malades de recevoir régulièrement et gratuitement des antirétroviraux. Ceci est confirmé par M. S. une personne infectée avec des propos suivants : "Depuis que je suis malade, je reçois gratuitement des médicaments qui retardent l'évolution de la maladie. Donc j'en prends souvent. Quand ça tend à finir, je renouvelle le stock à telle enseigne que je ne suis jamais en rupture ». En plus de la distribution d'antirétroviraux, les médecins suivent régulièrement les malades. Mieux l'infection du VIH/Sida est de nos jours considérée comme une maladie chronique. Grâce à l'efficacité des traitements antirétroviraux et à une prise en charge plus précoce, la maladie est mieux maîtrisée chez de nombreux patients. Ce qui fait que leur espérance de vie est proche de celle de la population générale. C'est ce que souligne K. A. patiente en ces termes : «Depuis que je suis déclarée séropositive, je suis suivie par un médecin qui me donne non seulement des antirétroviraux régulièrement mais aussi des conseils. Ces conseils me permettent de respecter mes rendez-vous à l'hôpital et de bien prendre mes 
médicaments. Ce qui fait que je n'ai pas trop de problèmes de santé ». En effet, les personnes vivant avec le VIH sont également exposées à un risque accru de co-infection c'est-à-dire vivre avec plus d'une infection à la fois. Pour prévenir au mieux cette co-infection, le suivi des personnes vivant avec le VIH doit être partagé et coordonné entre l'hôpital (à l'initiation du traitement et lors du contrôle annuel) et le médecin généraliste qui reste le professionnel de santé le plus consulté par les patients. C'est ce qu'évoque A. V. médecin généraliste pour soutenir cette idée en disant : "les malades et le personnel de santé sont obligés de travailler ensemble pour le bien être des patients. Si entre les deux entités la confiance est rompue alors il serait difficile d'être complices. Et cela ne facilitera la tâche des uns et des autres. Il faut aussi ajouter que l'hôpital constitue pour nous le lieu où nous devons nous voir régulièrement ». Toutefois, les différentes prises en charge se font non sans difficultés.

\section{Difficultés relatives à l'illettrisme des personnes infectées}

Dans la région du Moronou, l'enquête de terrain révèle qu'il ya plus analphabètes que de lettrés. Cela a un impacte considérable sur le traitement de la maladie. Car de nos jours le VIH/Sida se traite mais il ne se guérit pas. En effet, les antirétroviraux sont des comprimés qui se prennent à vie et à des heures précises. Ceci est confirmé par N.P. infirmier s'exprimant en ces termes : "Pour l'efficacité du traitement, les médicaments doivent être prises à des heures précises. Si par exemple le malade a l'habitude de prendre ses médicaments à huit heures du matin, il doit les prendre tous les jours à cette même heure. Sinon le médicament risque de ne pas être efficace. Mieux si le médicament doit être pris par deux fois dans la journée et que le malade oublie de respecter cette posologie. Alors, cela joue négativement sur le traitement. Et c'est des choses qui sont légion en zone rurale ». Ces situations arrivent souvent aux malades. En dehors de cet aspect, il ya la barrière linguistique. En effet, la plupart des malades ne parlent pas le français, ce qui fait qu'ils ont des problèmes à échanger avec le personnel médical. Dans ce cas, les médecins ou les infirmiers ont souvent recours à des interprètes qui ne sont pas forcement du corps. Ce qui n'est pas souvent du goût du malade. Cette idée a été soulevée par B.F. patiente en disant ceci : "Quand l'agent de santé veut échanger avec nous, il fait appel à quelqu'un de la région qui sert d'interlocuteur. Cela ne nous arrange pas car ce dernier peut nous exposer. Alors moi je veux que les médecins soient discrets pour nous protéger aussi, secret médical oblige »

Il faut aussi mentionner que la plupart des malades résident en zone rurale. Cependant les hôpitaux généraux et les centres de santé appropriés sont situés généralement en ville. C'est sur rendez-vous que les malades se rendent en ville pour avoir accès aux médicaments et aux soins médicaux. Pour y arriver, il faut débourser de l'argent pour faire le transport. La plupart des malades 
démunis n'y arrivent pas. Ce qui fait qu'ils ne respectent pas les rendez-vous. Cela joue naturellement sur leur traitement. Pour confirmer l'idée, Z.H. patiente dit ceci : "Mon village est situé à $15 \mathrm{Km}$ de la ville où se trouve l'hôpital général. C'est là-bas que se trouve mon médecin traitant. C'est donc lui qui me suit. Pour y aller, il me faut au minimum la somme deux milles francs. Et souvent pour l'avoir, c'est très difficile. Quand, le rendez-vous arrive et que je n'ai pas cette somme, je suis obligée de le griller. En effet, cela joue sur mon traitement ». A cause de la stigmatisation, les personnes vivant avec le VIH/Sida ne veulent pas que les autres membres de la communauté sachent qu'elles sont infectées par le virus même les autres malades de la même localité pour ne pas divulguer l'information. Cela rend difficile la prise en charge.

\section{Difficultés rencontrées pendant la prise en charge au niveau des structures impliquées et leur personnel}

\subsection{Au niveau des organisations non gouvernementales (ONG)}

Dans la région du Moronou, deux ONG se partagent la prise en charge des personnes infectées par le $\mathrm{VIH} /$ sida. Il s'agit de l'ONG ' 'tous pour le Toukpè 'située dans la ville de Bongouanou et de l'ONG',ACP2H (Action contre la pandémie VIH et autres handicapes, située quant à elle, à Arrah. Ces ONG s'occupent selon notre enquête 3587 patients. Elles rencontrent d'énormes difficultés quant à la prise en charge des malades.

Au niveau communautaire, le soutien psychologique et moral, se fait dans les villages au domicile des patients. Les organisations non gouvernementales rencontrent d'énormes difficultés. "Nous ne disposons d'aucun moyen de locomotion. Ce qui ne nous facilite pas la tache. Si par jour, nous devenons sillonner deux villages. Cela devient très difficile. La deuxième difficulté est que nous ne disposons pas de personnel en quantité et en qualité ». Avoue P. E. coordonnateur d'ONG. L'accompagnement psychologique échoit aux psychologues de formation. Mais les ONG ne disposant par de moyens conséquents pour pouvoir en embaucher, elles sont obligées de faire ce travail avec les moyens de bord. P.E. ajoute à cet effet « le peu de moyens que nous disposons avec les subventions reçues de nos partenaires, ne nous permettent d'engager et de payer un psychologue. Mieux de l'embaucher en plein temps $\gg$.

Les ONG déplorent le fait que dans les villages, elles ne disposent pas de représentants à cause de leur effectif limité. Ces derniers devaient être les relais. Ce qui faciliterait la prise en charge des personnes infectées par le VIH/Sida en milieu rural. Dans les villages, il n'existe pas de points focaux, ni de pairs éducateurs pour passer les informations au niveau du VIH/Sida dans la communauté. «Nous avons proposé de constituer de groupes dans les différents villages de la région mais les malades ont refusé catégoriquement 
cette proposition. Car selon eux, cela empêcherait la confidentialité de la maladie. Tout le village connaitra les malades du sida et la stigmatisation s'installera. Cela complique notre travail ». Révèle P. T. responsable d'ONG.

Concernant la prise en charge sociale des malades, les ONG rencontrent aussi des difficultés. Bien qu'elles assurent l'achat des ordonnances de certains patients mais les moyens dont elles disposent ne suffisent pas pour satisfaire tout le monde car la demande à ce niveau est très forte. A cela s'ajoute l'achat de lait pour les biberons de nouveaux nés. Car les femmes qui accouchent ne doivent pas donner leurs seins aux bébés pour ne les contaminer. Fort de cela, les ONG devraient distribuer des boites de lait aux patients nécessiteux.

\subsection{Au niveau des formations sanitaires et le corps médical : l'insuffisance des infrastructures médicales publiques, le manque de personnel formé}

La région du Moronou compte trois hôpitaux généraux : un hôpital général à Bongouanou, un hôpital général à Arrah et un hôpital général à M'batto. Elle compte 8 (huit) centres de santé urbains (Kangandisso, Andé, Assié Koumassi, N'guessankro, Kregbé, Abongoua, Tiémélékro, Anoumanba) et un dispensaire foyer de charité à Kotobi. Ces formations sanitaires s'occupent des personnes vivant avec le VIH/sida. Les 3587 patients partagent les services de ces formations sanitaires. Les formations sanitaires ne sont pas en nombre suffisant eu égard au nombre de malades qu'elles reçoivent par jour. Le personnel médical qui y travaille est en nombre insuffisant. Mieux ces structures sanitaires ne sont pas spécialisées au VIH/Sida. Elles s'occupent de toutes sortes de maladies. Ce qui fait que le personnel médical qui y travaille est généraliste, ce qui fait qu'il ne s'occupe pas spécifiquement du VIH/Sida. "Généralement, nous qui travaillons dans les structures sanitaires de la région, sommes des généralistes. Nous n'avons que quelques connaissances dans le domaine du sida. Ce qui fait qu'on ne peut que distribuer des médicaments(ARV) aux patients. Mais l'accompagnement psychologique nous échappe par moment ». Révèle M. G. médecin. De nous jours, les formations sur le VIH/sida se font rares. Et ces quelques rares formations sont souvent destinées aux médecins. Pour corroborer l'idée F. J. infirmier dit ce qui suit : « Nous sommes les lasser pour compte car les formations sont destinées aux cadres supérieurs de la santé en occurrence les médecins. Les infirmiers, les sages femmes et aides soignants se débrouillent avec quelques connaissances en matière du VIH/sida».

$\mathrm{Au}$ cours de l'enquête, la barrière linguistique a été soulignée par le personnel médical comme un frein à la prise en charge des malades dans la région du Moronou. Car la majorité de nos patients sont des analphabètes. Ainsi la communication qui devait s'établir directement entre le corps médical 
et les patients dans la région est bafouée. Le personnel médical ne comprenant pas la langue locale fait appel à une personne extérieure pour communiquer avec le malade. Ce qui constitue une violation au secret médical en vigueur en Côte d'Ivoire. "Un jour, j'ai eu la visite d'une vendeuse de pagne originaire de la région. Dans le même temps, je reçois une patiente qui ne comprenait pas le français, j'étais obligée de demander à la vendeuse de traduire à la dame ce que je devais lui dire. C'est ce qui s'est passé. C'est vrai que cette manière de faire n'est pas la bonne. Mais on est obligé de procéder ainsi si on veut que les choses avancent ». Nous confie Y. A. sage femme d'un centre de santé urbain de la région.

\section{Discussion et conclusion}

Cette étude a pour objectif d'analyser les différentes prises en charges reçues par les personnes vivant avec le VIH/Sida dans la région de Bongouanou et les difficultés rencontrées. Concernant l'hypothèse, ce travail de recherche a vérifié que le problème de la prise en charge des malades en milieu rural est lié à l'insuffisance des infrastructures de prise en charge, le manque de personnel formé et l'illettrisme des patients. Il ressort de cette étude que les personnes infectées par le VIH/Sida dans la région du Moronou bénéficient de deux types de prise en charge à savoir la prise en charge psychosociale et la prise en charge médicale ou thérapeutique. Ceci a été souligné par des auteurs tels que Zongo, Yeni, Toma et l'ONG Espoir de famille. Ainsi, selon Zongo et al. (2009), au Benin, en plus des mesures adoptées pour la prévention de la transmission du VIH au niveau de la population, plusieurs stratégies ont été mises sur pieds pour assurer l'accompagnement des personnes infectées par le virus du sida. C'est pourquoi, ces personnes là ont besoin d'être soutenues pour le relever les défis de cette maladie chronique qui suscite le rejet de la société. Le bouleversement dès l'annonce et vécu de la maladie et surtout l'incidence de ses complications psychosociales nécessitent une prise en charge thérapeutique et psychosociale. Selon Perla (2012), en ce qui concerne la prise en charge des individus infectés par le VIH/Sida, elle ne s'arrête pas seulement à l'accès aux médicaments et aux soins médicaux mais il faut ajouter un soutien psychosocial et spirituel. Pour Yeni (2006), la prise en charge doit être thérapeutique, psychologique, préventif et social. Avant de débuter un traitement ARV, un bilan précis, clinique, immuno-virologique, métabolique et des facteurs de risque cardiovasculaire est essentiel et le patient doit être informé des objectifs du traitement, de la nature, de la fréquence et de la gravité potentielle des effets indésirables. Il faut donc souligner que la prise en charge des personnes vivant avec le VIH/Sida de manière générale est très complexe particulièrement en province. Pour l'ONG Espoir de la famille (2014), l'accompagnement psychosocial à base communautaire des personnes infectées par le VIH/Sida 
et des orphelins du Sida, leur apporte un réconfort moral et mental en améliorant leurs conditions de vie. Selon Toma et al. (2013), malgré le prolongement de l'espérance de vie des personnes infectées par le VIH/Sida dû au traitements antirétroviraux, l'atteinte psychologique persiste et doit être comme une partie de la prise en charge afin qu'elle soit totale et efficace. En somme, les résultats de cette étude confirment l'orientation des écrits antérieurs sur la question. En effet, les personnes vivant avec le VIH/sida dans la région du Moronou bénéficient de deux types de prise en charge à savoir les prises en charge psychosociale et médicale.

Ces prises en charge se font cependant avec difficultés. Les résultats ont également montré que pendant la prise, des difficultés ont été rencontrées tant au niveau des ONG qu'au niveau des formations sanitaires. Les difficultés sont relatives à l'illettrisme des personnes infectées, à l'insuffisance des structures impliquées dans la prise en charge et surtout le personnel formé. Concernant l'illettrisme des patients, il faut indiquer qu'il constitue un frein dans leur prise en charge. Cela a été soulevé par des écrits antérieurs. C'est le cas de l'Association médicale mondiale. En effet, selon Association médicale mondiale (2006), le VIH/SIDA constitue non seulement de nos jours, un véritable problème de santé publique mais aussi celui de droits humains. Les facteurs comme la pauvreté, l'absence de domicile, l'illettrisme, la prostitution, la traite d'êtres humains, la toxicomanie, la stigmatisation, la discrimination et l'inégalité des sexes contribuent à propager la maladie. Il en est de même pour les facteurs sociaux, économiques, juridiques et ceux ayant trait aux droits humains qui ont un impact sur l'aspect sanitaire et les relations/ les décisions du personnel de santé et les malades. Le manque de ressources humaines et financières dans systèmes de santé ont un impact négatif sur les efforts de lutte contre cette maladie.

Pour des auteurs comme Akrich, Grimaldi, Rabeharisoa, Delbanco, Massé et Jouet ont placé le patient au centre de la lutte contre le sida. Donc, son implication est très déterminante. C'est pour quoi une faille de sa part joue sur la prise en charge. Pour ces auteurs, cette intervention de la personne infectée par le VIH/Sida entant qu'acteur principal dans la lutte contre le Sida. Elle se trouve dans processus de participation profane pour une démocratie de la santé (Akrich et al., 2009 ; Grimaldi, 2010 ; Akrich \& Rabeharisoa, 2012) basée sur l'implication de tous les acteurs socio-économiques et politiques dans la problématique du développement de la santé. Cette implication du patient est une question pour situer l'apport de l'expertise profane dans la construction des savoirs sur la maladie (Delbanco et al., 2001 ; Massé, 1995 ; Jouet et al., 2010).

Concernant, l'insuffisance des structures de prise en charge et le manque de personnel qualifié et bien formé dans la région du Moronou, les résultats de terrain l'attestent bien. Les auteurs Meessen, Van Damme, 
Lefeuvre abondent dans le même sens à travers des écrits. Selon Meessen et Van Damme (2005). Les pays à faible revenu d'Afrique et d'Asie font face à de nombreux défis sanitaires. Les services de santé en place aujourd'hui y répondent avec peine. L'insuffisance de moyens a des conséquences tragiques. Sans conteste, un effort accru du Nord est nécessaire.

Toutefois, la faible performance de ces systèmes de santé mérite également une attention critique. Si les institutions actuelles mises en place sont défectueuses, des reformes s'avèrent nécessaires. Pour Lefeuvre et al. (2014), les pays pauvres où le VIH/Sida est une charge, avec un manque de personnel qualifié pour une prise en charge de qualité aux personnes touchées par la maladie. Cela est dû au fait que, bon nombre de professionnels de santé quitte leur pays d'origine après leur formation pour des destinations plus attractives. Ceux qui restent travaillent dans des situations précaires avec une baisse de rendement. Par ailleurs, on assiste à une disparité dans la répartition des professionnels de santé pour laquelle les zones rurales sont les plus lésées.

De tout ce précède, nous pouvons affirmer que les différentes formes de prises en charge bénéficiées par patients du VIH/Sida dans la région du Moronou sont psychosociale et thérapeutique. Mais au niveau de ces prises en charge, l'on rencontre de difficultés qui sont l'illettrisme des patients, l'insuffisance des structures de prise en charge et le manque de personnel qualifié et bien formé.

\section{References:}

1. Akrich, M., Meadel, C., \& Rabeharisoa, V. (2009). Se mobiliser pour la santé, des associations de patients témoignent. Collection Sciences socialesParis-Tech.DOI : 10.4000.

2. Akrich, M. \& Rabeharisoa, V. (2012). «L'expertise profane dans les associations de patients, un outil de démocratie sanitaire », Santé publique,24/1:69-74DOI : 10.3917.

3. Association AFIA (2016). Prise en charge des personnes vivant avec le VIH/sida. http://www.afia-fev.org/prise-en-charge-des-personnesvivant-avec-le-vihsida/. Consulté le 17 mai 2019.

4. Association médicale mondiale (2006). Prise de Position de l'AMM sur le VIH/SIDA et la Profession Médicale. Adoptée par la $57^{\mathrm{e}}$ Assemblée générale de l'AMM, Pilanesberg, Afrique du Sud, octobre 2006 et amendée par la $68^{\text {ème }}$ Assemblée générale, Chicago, EtatsUnis, Octobre 2017.https://www.wma.net/fr/policies-post/prise-deposition-de-lamm-sur-le-vihsida-et-la-profession-medicale. Consulté le 10/03/2019.

5. Balint, M. (1957). The doctor, his patient and the illness, London, PITMAN.DOI : 10.1097/00000441-195711000-00018

6. Barbot, J. (2002). Les malades en mouvements, Paris, Balland. 
7. Delfraissy, J. F. (2002). Prise en charge thérapeutique des personnes infectées par le VIH. Rapport. Paris : Flammarion : Médecine-Sciences (éditeur).

8. Desclaux, A. (2003). VIH/SIDA stigmatisation et discrimination : une approche anthropologique. Actes de la table ronde organisée le 29 novembre 2002 à l'UNESCO - Paris. Études et rapports, série spéciale, $\mathrm{n}^{\circ} 20$ Division des politiques culturelles et du dialogue interculturel UNESCO.

9. Delbanco, D., Berwick, M., Boufford, J-I., Edgman-Levitan, S., Ollenschlaèger, G., Plamping, D. \& Rockefeller, G. (2001). "Health care in a land called people power: Nothing about me without me", Health expectations, 4: 144-150.

10. Eboko, F., Abe, C. \& Christian, L. (2010). Accès décentralisé au traitement du VIH/Sida, Evaluation de l'expérience camerounaise. Paris, ANRS, Collection Sciences sociales.

11. Epstein, S. (2001). Histoire du sida. 2. La grande révolte des malades, Paris, Les Empêcheurs de penser en rond.

12. Grimaldi, A. (2010). «Les différents habits de l'expertise profane », Presses de Sciences Po, vol 2 n$^{\circ} 27$ : 91-100.

13. Hanna, G. (1995). Wilderness-related environmental outcomes of adventure and ecology education programming. The Journal of Environmental Education, 27(1), 21-32.

14. Heba A. (2009). The New Humanitarian Accéder aux services $\mathrm{VIH} / \mathrm{SIDA}$ en zone rurale.

https://www.thenewhumanitarian.org/content/information-sur-leservice-d-actualites-francophones-produit-par-new-humanitarianautrefois. Consulté 17 mai 2019.

15. Jouet, E., Flora, L. \& Las Vergnas, O. (2010). Construction et reconnaissance des savoirs expérientiels des patients: note de synthèse, [en ligne], http://www-ufr8.univ-paris8.fr/pfa/textpdf/5859synthese_sante.pdf, (page consultée le 29/01/2013).

16. Lefeuvre, D., Dieng, M., Lamara, F., Raguin, G. \& Michon, C. (2014). Les agents de santé communautaire dans la prise en charge thérapeutique du VIH/sida. Santé Publique (Vol. 26) no 6. P. 879 à 888. https://www.cairn.info/revue-sante-publique-2014-6-page879.htm. Consulté le 10/03/2019.

17. Masse, R. (1995). Culture et santé publique, Montréal, Gaétan Morin.

18. Mc Cormack Brown, K. (1999a). Theory of reasoned action / Theory of planned Behavior. Disponible : http://hsc.usf.edu/ kmbrown/TRA_TPB.htm 
19. Mc Laughlin, H. (2009). "What's in a name: 'client', 'patient', 'customer', 'consumer', 'expert by experience', 'service user', what else's next?" British journal of social work, 39/9 : 1101-1117.

20. Meessen, B. \& Van Damme, W. (2005). Systèmes de santé des pays à faible revenu : vers une révision des configurations institutionnelles ? Mondes en développement2005/3 ( $\mathrm{n}^{\mathrm{o}}$ 131). P. 59 à 73. https://www.cairn.info/revue-mondes-en-developpement-2005-3page-59.htm. Consulté le 10/03/2019.

21. Mounsade, K. F. (2012). « Accès aux antirétroviraux et prise en charge du sida au Cameroun » in MENGUE M-T. (dir.), Développement et changement social en Afrique, Yaoundé, Presses de l'UCAC : 249262.

22. N'cho, S.D., Issaka, T., Bénie, J. B. V., Diaby, B., Zengbé-Acray, P., Attoh-Touré, H., Adjoua, D., Ekra, K.D. \& Tagliante-Saracino, J. (2013). Séroprévalence de l'infection à VIH en conseil et dépistage volontaire par stratégie mobile en Zone rurale en Côte d'Ivoire. Santé Publique 2013/6 (Vol. 25), pages 849 à 856.

23. OMS (1998). Education thérapeutique du patient. Genève : OMS.

24. ONG Espoir de la famille (2014). Prise en charge psychologique et sociale des PVVIH et des OEV.

25. https://www.espoirdelafamille.org/prise-en-charge-psychologique-etsociale-des-pvvih-et-des-oev. Consulté le 08/03/2019.

26. ONUSIDA (2000). Education par les pairs et VIH/Sida : concepts utilisations et défis, Genève : ONUSIDA.

27. ONUSIDA (1997a). Conseil et VIH/Sida : Actualisation ONUSIDA, Collection ONUSIDA, les meilleures pratiques. Genève : ONUSIDA.

28. ONUSIDA (1997b). Mobilisation communautaire et sida Collection Meilleures pratiques [en ligne], http://data.unaids.org/publications/ircpub03/commmob-tu_fr.pdf, (page consultée le 13/02/ 2010).

29. Parsons, T. (1951). The social system, Glencoe, Free Press.

30. Perla, B. (2012). Problématique de la prise en charge des personnes vivant avec le virus du sida de l'hôpital général de Goma en RDC. Institut supérieur de développement rural des grands lacs Goma.

31. Pierron, J-P. (2007). «Une nouvelle figure du patient? Les transformations contemporaines de la relation de soins », Sciences Sociales et Santé, 25/2: 43-66. DOI : 10.3917.

32. Plan National Stratégique (2000/2005). Instituant la prise en charge hospitalière du VIH/sida n'apporte aucune précision sur la nature et le contenu de la formation dont il est question.

33. Ruffié, J. \& Sournia, J.C. (1995). Les épidémies dans l'histoire de l'homme. Paris, Flammario 
34. Stepanoff, S. (2010). Les entreprises dans la lutte contre le VIH/sida. Direction du Développement de l'association SIDA-ENTREPRISES*.

35. Thani, M. S. (2014). Lutte contre le sida et prise en charge des personnes vivant avec le virus du sida à Mayotte , $14^{\text {e }}$ législature Question orale ${ }^{\circ}$ 0808S (Mayotte - SOC) publiée dans le JO Sénat page 1285.

36. Toma, S., Sartori, M., Weber, K., Canuto, A., Moayedoddin, B., Trellu, L.T., Calmy, A., Pichard, C. \& Joly, C. (2013). Bénéfices d'un accompagnement psychologique des patients vivant avec le VIH. Rev Med Suisse 2013; volume 9. 374-377

37. Villarreal, M. (2014). Chargée de liaison de la FAO pour le VIH/SIDA Paru le :25févr.2014. http://www.fao.org/Focus/F/aids/aids6-f.htm, consulté le 16 mai 2019.

38. Weller, J. (2003). «L'inventivité militante, le sida et les nouveaux traitements », Sciences Sociales et Santé, 21/3 : 37-70.

39. Yeni, P. (2006). Les nouvelles recommandations de prise en charge des personnes infectées par le VIH. Journal du sida no189.

40. Zongo, M., Capochichi, J., Gandaho, P. \& Coppieters, Y. (2009). Prise en charge psychosociale des personnes vivant avec le VIH au Bénin. Santé Publique, Vol. 21 no 6, P 631-639. 